\title{
Assessment of hormonal levels as prognostic markers and of their optimal cut-offs in small intestinal neuroendocrine tumours grade 2
}

\author{
Dimitrios Papantoniou $\mathbb{1 0}^{1,2} \cdot$ Malin Grönberg ${ }^{1} \cdot$ Kalle Landerholm ${ }^{3} \cdot$ Staffan Welin ${ }^{1}$ Barbara Ziolkowska ${ }^{4}$. \\ Dennis Nordvall ${ }^{5}$ Eva Tiensuu Janson ${ }^{1}$
}

Received: 6 June 2020 / Accepted: 24 October 2020 / Published online: 26 November 2020

(c) The Author(s) 2020

\begin{abstract}
Purpose Small intestinal neuroendocrine tumours (siNETs) with a Ki-67 proliferation index between 3 and $20 \%$ belong to WHO grade 2. Response to treatment may be monitored by blood chromogranin A $(\mathrm{CgA})$ and urine 5-hydroxyindoleacetic acid (5HIAA). The aim of this retrospective study was to investigate the prognostic value of baseline CgA and 5HIAA and of the early biochemical response to treatment, and to compare different cut-off values used in the literature.

Methods A retrospective cohort study of 184 patients with siNET Grade 2 treated with somatostatin analogues (SSA), interferon-alpha (IFN) or peptide receptor radionuclide therapy (PRRT).

Results Baseline CgA was a statistically significant prognostic marker for both cancer-specific survival (CSS) and progression-free survival (PFS). A cut-off of $5 \times$ ULN (upper limit of normal) was best discriminative in most cases, but $2 \times$ ULN discriminated better for SSA. Baseline 5HIAA was a prognostic marker for CSS in treatment with IFN and PRRT, but not for single SSA. Early changes of CgA and 5HIAA correlated well with CSS (HR 3.18, 95\% CI 1.82-5.56 and HR 1.47, 95\% CI 1.16-1.86) and PFS (HR 3.08, 95\% CI 1.86-5.10 and HR 1.37, 95\% CI 1.11-1.68) for SSA, but not for PRRT. Conclusions Baseline CgA and to a lesser extent 5HIAA are associated with CSS irrespective of treatment used, and with PFS after PRRT, and $5 \times$ ULN provides best discrimination in many, but not all, cases. Early reductions of CgA and 5HIAA are prognostic for treatment with SSA, but not PRRT.
\end{abstract}

Keywords Chromogranin A $\cdot 5$ HIAA $\cdot$ Small intestinal neuroendocrine tumours $\cdot$ Biomarker $\cdot$ Carcinoids $\cdot$ Cut-offs

Supplementary information The online version of this article (https:// doi.org/10.1007/s12020-020-02534-8) contains supplementary material, which is available to authorized users.

Dimitrios Papantoniou

dimitris.papantoniou@medsci.uu.se

1 Department of Medical Sciences, Endocrine Oncology, Uppsala University, Uppsala, Sweden

2 Department of Oncology, Ryhov County Hospital, Jönköping, Sweden

3 Department of Surgery, Ryhov County Hospital, Jönköping, Sweden

4 Maria Sklodowska-Curie National Research Institute of Oncology, Gliwice, Poland

5 Qulturum, Region Jönköping, Jönköping, Sweden

\section{Introduction}

Neuroendocrine neoplasms (NENs) of the small intestine are the third largest subgroup of NENs in the gastroenteropancreatic system [1]. According to the WHO classification from 2019, they are grouped on the basis of their proliferation index into well differentiated grade 1 (G1, $\mathrm{Ki}-67<3 \%)$, grade $2(\mathrm{G} 2, \mathrm{Ki}-673-20 \%)$ and grade $3(\mathrm{G} 3$, $\mathrm{Ki}-67>20 \%$ ) neuroendocrine tumours (NETs) and the rare, poorly differentiated G3 neuroendocrine carcinomas (NEC, Ki-67>20\%) [2]. In a recent Surveillance Epidemiology and Ends Results database analysis, which uses an older slightly different classification system, well differentiated NETs were approximately four times more common than intermediate grade tumours [3]. NETs G2 have a more aggressive clinical behaviour than their G1 counterparts and probably altered molecular background [4].

Two monoanalytes, plasma chromogranin $\mathrm{A}(\mathrm{CgA})$ and urine 5-hydroxyindoleacetic acid (5HIAA) are used to monitor the course of these neoplasms during treatment of 
non-curable tumours, and may also have a role in detection of recurrence after potentially curative surgery or other treatments [1]. CgA has been studied for two decades as a potential diagnostic marker for NENs. However, more recent work has failed to support this idea, and a recent Delphi consensus concluded that no single biomarker meets the sensitivity and specificity standards to be considered as a diagnostic tool $[5,6]$. Although conflicting evidence exists regarding their prognostic and predictive value, most studies report that at least higher $\mathrm{CgA}$ levels at baseline are associated with shorter survival, while significance of $\mathrm{CgA}$ changes for predicting worse response to treatment is still unclear. [7-9]. When dichotomizing these biomarkers, different authors have been using various cut-offs ranging from 1 to 10 times the upper limit of normal $(\times \mathrm{ULN})$, leading to results that are not entirely comparable [7, 10-16]. Limited data shows that early reduction of $\mathrm{CgA}$ and/or 5HIAA may correlate with treatment effect of some, but not all anti-tumoural agents, and with the survival of the patient $[17,18]$.

Despite differences between G1 and G2 small intestinal NETs (siNETs), most studies to date have examined them as a uniform group, and often in combination with NETs of other origin. Their results are thus more representative of the much more common G1 tumours. To the best of our knowledge, this is the first study investigating the association between baseline $\mathrm{CgA}$ and 5HIAA, as well as their change during treatment, in a uniform cohort of patients with G2 tumours of the small intestine, treated with somatostatin analogues (SSA), peptide receptor radionuclide therapy (PRRT) and/or interferon-alpha (IFN).

\section{Materials and methods}

In this retrospective cohort study, all patients with metastatic siNETs with a Ki-67 proliferation index between 3 and $20 \%$ (WHO grade 2) that were treated at the Department of Endocrine Oncology, Uppsala University Hospital, a tertiary referral centre, and at the Department of Oncology, Ryhov County Hospital, a regional hospital, between 1 January 2000 and 31 May 2017 were eligible for inclusion and were retrieved from an internal database. Patients treated by surgery after initiation of cancer-specific treatment, with no evidence of remaining disease and no recurrence during the study period, were excluded from the survival analysis. Following approval from the Uppsala ethical review board (Dnr 2017/403), data on patients' clinical status, treatments given and laboratory tests were extracted from the hospitals' medical archives. Medical records were re-reviewed to determine cause of death and survival status was censored on 31st October 2019 or at last known contact. Causes of death due to tumour progression, adverse events, surgical morbidity as well as cases where cause of death was indeterminate but cancer-related death was likely, were classified as cancer-specific mortality. Patients dying from other causes, not related to their NET tumour, were censored at time of death. Cancer-specific survival (CSS) and progression-free survival (PFS) were calculated for each treatment given. Progression was defined as radiological progression, according to multidisciplinary team assessment, whenever available. It is worth noting that RECIST criteria were not consistently applied during the study period, and that in a small subset of mostly older patients (less than 10\%) treating physician's assessment of biochemical/clinical progression was accepted as time for progression.

Biomarkers were collected at baseline, and at the 6-month visit. In a minority of patients undergoing interventional procedures within this 6-month period, last biomarker control before intervention was accepted, as long as it was at least 3 months after treatment start; otherwise these patients were excluded from analysis. For the measurement of 5HIAA, patients were provided with one or two receptacles and were asked to collect urine for one or two 24-h periods prior to the planned visit and to maintain a serotonin-poor diet for $72 \mathrm{~h}$ beforehand. 5HIAA was measured as a single sample or as two samples on consecutive days; whenever two samples were examined, the mean value was used. Samples were analyzed using highperformance liquid chromatography. Plasma samples for CgA were collected in chilled heparinised vacutainer tubes after fasting overnight. All samples before and during PRRT were measured at Uppsala University Hospital using the EuroDiagnostica kit (Malmö, Sweden) for CgA. Samples before and during other treatments were measured at Uppsala University Hospital (85\% of evaluable samples for patients treated with SSA or IFN) or at the patient's local laboratory. In each case, baseline and 6-month tests were conducted at the same laboratory. Levels of $\mathrm{CgA}$ and 5HIAA were described as $\times \mathrm{ULN}$ of the reporting laboratory.

Biochemical partial response (PR) was defined as a reduction of baseline $\mathrm{CgA}$ or $5 \mathrm{HIAA}$ by at least $50 \%$ and biochemically progressive disease (PD) as an increase by at least $25 \%$. Patients with values at 6 months between $-50 \%$ and $+25 \%$ of baseline were deemed as having biochemically stable disease (SD).

\section{Statistical methods}

Statistical analysis was performed with $\mathrm{R}$ version 3.5 .3 , and the compareGroups package 4.0.0, using standard methodology (chi-square test for dichotomous variables, $t$-test for continuous variables and semi-parametric cox models for censored variables). PFS and CSS were analyzed using the Kaplan-Meier method and between-group differences were 
analyzed using a log-rank test. Hazard ratios (HRs) and confidence intervals (CIs) were estimated from the Cox proportional hazards model. Optimal cut-off points were calculated with R packages Survminer 0.4.3 and maxstat 0.7-25 using the maximally selected rank statistics, a method that allows the evaluation of cut-off points, which provide the classification of different risk groups in a quantitative or ordered predictor variable $[19,20]$. Sensitivity, specificity, positive (PPV) and negative predictive values (NPV) were estimated with R package timeROC 0.3 .

All tests were two-sided. $P$ values $<0.05$ were considered statistically significant.

\section{Results}

\section{Demographics}

In total, 184 patients with metastatic siNET G2 were included in the present study. During the study period 182 patients were treated with SSA, 93 patients with IFN and 92 patients with PRRT. Four patients were rechallenged with IFN and 13 with PRRT, and were included as separate treatment events. Additional treatments including chemotherapy and everolimus were offered to less than $20 \%$ of patients and were not analyzed. Most patients started treatment with SSA. PRRT was used mostly as second or third line of treatment. The patterns of use of IFN changed significantly during the study period: During the initial years, it was used either as single first line treatment (13 patients) or in parallel with SSA, often starting at the same time or within a few months of SSA initiation $(62 \%$ of the IFN treatment cases). During the latter phase of the study IFN was mostly used as second or third line treatment. Baseline biochemical markers and selected demographics are summarized in Table 1. A consort diagram is shown in Fig. 1.

\section{Baseline biomarkers as prognostic markers}

We examined the association between biomarker $(\mathrm{CgA}$ and 5HIAA) levels at treatment start and survival (CSS and PFS) both as continuous and dichotomous variables. Baseline $\mathrm{CgA}$ was consistently a statistically significant prognostic marker for both CSS and PFS as a continuous variable (non-significant association in the case of PFS on SSA) (Table 2). Baseline 5HIAA was a statistically significant prognostic marker for CSS in patients treated with IFN and PRRT, but not in patients treated with single SSA, and a statistically significant prognostic marker of PFS for patients treated with PRRT (Table 3).

Using the R package Survminer, we estimated optimal cut-off points for each treatment and for the whole cohort, irrespective of treatment. The estimated optimal cut-offs for different treatments varied considerably (Table 4). For the
Table 1 Baseline patient characteristics

\begin{tabular}{|c|c|c|c|}
\hline & $\begin{array}{l}\text { SSA } \\
(N=182)\end{array}$ & $\begin{array}{l}\text { IFN } \\
(N=97)\end{array}$ & $\begin{array}{l}\text { PRRT } \\
(N=105)\end{array}$ \\
\hline \multicolumn{4}{|l|}{ Sex } \\
\hline Female & $73(40.1 \%)$ & $40(41.2 \%)$ & $40(38.1 \%)$ \\
\hline Male & $109(59.9 \%)$ & $57(58.8 \%)$ & $65(61.9 \%)$ \\
\hline $\begin{array}{l}\text { Age at start } \\
\text { (median [IQR]) }\end{array}$ & $64.7[58.3 ; 71.5]$ & $60.9[53.7 ; 68.2]$ & $65.9[58.4 ; 71.1]$ \\
\hline \multicolumn{4}{|l|}{$\begin{array}{l}\text { Resection of } \\
\text { primary tumour/ } \\
\text { lymph nodes }\end{array}$} \\
\hline No & 47 (26.4\%) & $20(20.8 \%)$ & $20(19.2 \%)$ \\
\hline Yes & $131(73.6 \%)$ & $76(79.2 \%)$ & $84(80.8 \%)$ \\
\hline \multicolumn{4}{|l|}{ Metastasectomy } \\
\hline No & $122(67.0 \%)$ & $56(57.7 \%)$ & $64(61.0 \%)$ \\
\hline Yes & $60(33.0 \%)$ & $41(42.3 \%)$ & $41(39.0 \%)$ \\
\hline \multicolumn{4}{|l|}{$\begin{array}{l}\text { Performance } \\
\text { status (WHO) }\end{array}$} \\
\hline 0 & $65(58.0 \%)$ & $34(58.6 \%)$ & $52(54.7 \%)$ \\
\hline 1 & $31(27.7 \%)$ & $15(25.9 \%)$ & $33(34.7 \%)$ \\
\hline 2 & $12(10.7 \%)$ & $8(13.8 \%)$ & $9(9.5 \%)$ \\
\hline$\geq 3$ & $4(3.6 \%)$ & $1(1.7 \%)$ & $1(1.1 \%)$ \\
\hline $\begin{array}{l}\text { Ki-67 } \\
\text { (median [IQR]) }\end{array}$ & $7 \%[4 \% ; 10 \%]$ & $6 \%[4 \% ; 10 \%]$ & $8 \%[5 \% ; 12 \%]$ \\
\hline \multicolumn{4}{|l|}{$\begin{array}{l}\text { Bone/lung } \\
\text { metastases }\end{array}$} \\
\hline No & $114(75.5 \%)$ & $50(67.6 \%)$ & $49(49.0 \%)$ \\
\hline Yes & $37(24.5 \%)$ & $24(32.4 \%)$ & $51(51.0 \%)$ \\
\hline \multicolumn{4}{|l|}{ Line of treatment } \\
\hline 1 & $168(93.3 \%)$ & $60(62.5 \%)$ & $9(8.7 \%)$ \\
\hline 2 & $12(6.7 \%)$ & $29(30.2 \%)$ & $60(57.7 \%)$ \\
\hline$\geq 3$ & $0(0.0 \%)$ & $7(7.3 \%)$ & $35(33.7 \%)$ \\
\hline \multicolumn{4}{|l|}{$\begin{array}{l}\text { Concomitant start } \\
\text { SSA/other }\end{array}$} \\
\hline No & $112(63.3 \%)$ & $35(41.7 \%)$ & $96(91.4 \%)$ \\
\hline Yes & $65(36.7 \%)$ & $49(58.3 \%)$ & $9(8.6 \%)$ \\
\hline $\begin{array}{l}\text { Baseline CgA, × } \\
\text { ULN } \\
\text { (median [IQR]) }\end{array}$ & $6.8[2.1 ; 34.0]$ & $8.0[2.2 ; 45.2]$ & $16.5[4.5 ; 32.0]$ \\
\hline \multicolumn{4}{|l|}{$\begin{array}{l}\text { Baseline } \mathrm{CgA} \text {, } \\
\text { dichotomized }\end{array}$} \\
\hline$<5$ & $49(40.5 \%)$ & $31(43.7 \%)$ & $27(26.7 \%)$ \\
\hline $5-10$ & $17(14.0 \%)$ & $6(8.5 \%)$ & $12(11.9 \%)$ \\
\hline$>10$ & $55(45.5 \%)$ & $34(47.9 \%)$ & $62(61.4 \%)$ \\
\hline $\begin{array}{l}\text { Baseline 5HIAA, × } \\
\text { ULN } \\
\text { (median [IQR]) }\end{array}$ & $4.6[1.3 ; 15.5]$ & $4.2[1.1 ; 15.0]$ & $6.1[2.7 ; 15.0]$ \\
\hline \multicolumn{4}{|l|}{$\begin{array}{l}\text { Baseline 5HIAA, } \\
\text { dichotomized }\end{array}$} \\
\hline$<5$ & $68(55.3 \%)$ & $40(58.0 \%)$ & $42(41.6 \%)$ \\
\hline $5-10$ & $16(13.0 \%)$ & $7(10.1 \%)$ & $24(23.8 \%)$ \\
\hline$>10$ & $39(31.7 \%)$ & $22(31.9 \%)$ & $35(34.7 \%)$ \\
\hline
\end{tabular}

SSA somatostatin analogues, IFN Interferon-alpha, PRRT peptide receptor radionuclide therapy, $C g A$ chromogranin A, 5HIAA 5-hydroxyindoleacetic acid, $U L N$ upper limit of normal, $I Q R$ interquartile range

group as a whole, suggested cut-offs were $6 \times$ ULN of both $\mathrm{CgA}$ and 5HIAA for CSS. Respective cut-offs for PFS were $6 \times \mathrm{ULN}$ and $5 \times \mathrm{ULN}$.

Subsequently, we calculated HRs for CSS and PFS at "standard" dichotomized cut-off values used in the literature 


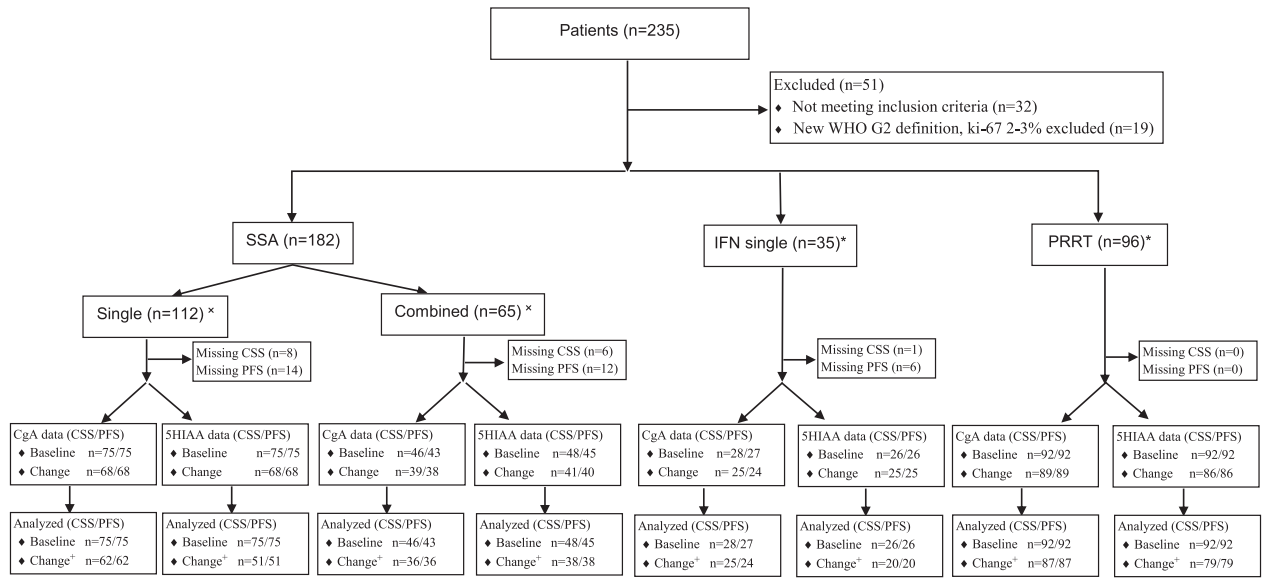

Fig. 1 SSA somatostatin analogues, IFN interferon, PRRT peptide receptor radionuclide therapy, $\mathrm{CgA}$ Chromogranin A, 5HIAA 5hydroxyindoleacetic acid, CSS cancer-specific survival, PFS progression-free survival, 5HIAA 5-hydroxyindoleacetic acid. ${ }^{\times}$Five patients with uncertain status excluded. *Treatments given. Most patients received several lines of treatment. Four patients were rechallenged with IFN and 13 with PRRT, and were included as separate treatment events. Approximately one third of patients treated with SSA and IFN had missing baseline and/or 6-month $\mathrm{CgA}$ and 5HIAA data. ${ }^{+}$For SSA single, SSA combined, IFN, and PRRT, 6, 3, 0 and 2 cases with baseline normal $\mathrm{CgA}$ and 17, 3, 5 and 7 cases with normal baseline 5HIAA were excluded from early biochemical response analysis

Table 2 Baseline CgA as predictor for CSS, PFS

\begin{tabular}{|c|c|c|c|c|c|c|c|c|}
\hline & \multicolumn{2}{|l|}{ SSA single } & \multicolumn{2}{|l|}{ SSA combined } & \multicolumn{2}{|l|}{ IFN } & \multicolumn{2}{|l|}{ PRRT } \\
\hline & $\mathrm{HR}[95 \% \mathrm{CI}]$ & $p$ & $\mathrm{HR}[95 \% \mathrm{CI}]$ & $p$ & $\operatorname{HR}[95 \% \mathrm{CI}]$ & $p$ & $\operatorname{HR}[95 \% \mathrm{CI}]$ & $p$ \\
\hline \multicolumn{9}{|l|}{ CSS } \\
\hline & $N=75$ & & $N=46$ & & $N=28$ & & $N=92$ & \\
\hline Continuous & $1.008[1.003 ; 1.012]$ & $<0.01$ & $1.008[1.004 ; 1.011]$ & $<0.01$ & $1.012[1.004 ; 1.020]$ & $<0.01$ & $1.016[1.008 ; 1.023]$ & $<0.01$ \\
\hline $5-10 x$ & & 0.02 & & 0.10 & & 0.02 & & $<0.01$ \\
\hline $5-10 \times$ & $1.39[0.49 ; 3.97]$ & & $0.85[0.22 ; 3.22]$ & & $3.37[0.56 ; 20.28]$ & & $5.90[1.97 ; 17.66]$ & \\
\hline$>10$ & $2.78[1.32 ; 5.87]$ & & $2.12[0.95 ; 4.75]$ & & $6.01[1.56 ; 23.21]$ & & $9.06[3.75 ; 21.88]$ & \\
\hline$>2$ & $5.30 * * *[1.85 ; 15.21]$ & $<0.01$ & $1.49[0.61 ; 3.67]$ & 0.38 & $2.65[0.58 ; 12.05]$ & 0.19 & NA & \\
\hline$>5$ & $2.27[1.12 ; 4.62]$ & 0.02 & $1.76[0.80 ; 3.87]$ & 0.15 & $5.15 *[1.40 ; 18.99]$ & 0.01 & $8.44 * * *[3.51 ; 20.31]$ & $<0.01$ \\
\hline$>10$ & $2.55[1.29 ; 5.01]$ & 0.01 & $2.21 * * *[1.06 ; 4.64]$ & $\mathbf{0 . 0 3}$ & $4.30[1.37 ; 13.51]$ & 0.01 & $4.56[2.45 ; 8.50]$ & $<0.01$ \\
\hline$>$ Optimal: treatment & $5.30 * * *[1.85 ; 15.21]$ & $<0.01$ & $1.49[0.61 ; 3.67]$ & 0.38 & $23.88 * * *[4.47 ; 127.62]$ & $<0.01$ & $8.32[2.97 ; 23.25]$ & $<0.01$ \\
\hline$>$ Optimal & $2.88[1.43 ; 5.82]$ & $<0.01$ & $1.71[0.81 ; 3.61]$ & 0.15 & $5.15[1.40 ; 18.99]$ & 0.01 & $6.38[2.94 ; 13.85]$ & $<0.01$ \\
\hline \multicolumn{9}{|l|}{ PFS } \\
\hline & $N=75$ & & $N=43$ & & $N=27$ & & $N=92$ & \\
\hline Continuous & $1.002[0.998 ; 1.006]$ & 0.35 & $1.004[1.002 ; 1.007]$ & $<0.01$ & $1.005[1.000 ; 1.010]$ & 0.04 & $1.007[1.003 ; 1.011]$ & $<0.01$ \\
\hline $5-10 x$ & & 0.29 & & 0.36 & & 0.03 & & $<0.01$ \\
\hline $5-10 x$ & $1.64[0.79 ; 3.39]$ & & $0.88[0.27 ; 2.83]$ & & $6.78[1.02 ; 45.11]$ & & $3.88[1.13 ; 13.29]$ & \\
\hline$>10$ & $1.45[0.82 ; 2.57]$ & & $1.61[0.74 ; 3.48]$ & & $4.62[1.22 ; 17.55]$ & & $6.36[2.58 ; 15.72]$ & \\
\hline$>2$ & $2.22 * * *[1.21 ; 4.07]$ & 0.01 & $0.94[0.40 ; 2.18]$ & 0.87 & $4.24[0.54 ; 33.00]$ & 0.14 & $4.01[0.96 ; 16.80]$ & 0.04 \\
\hline$>5$ & $1.50[0.89 ; 2.55]$ & 0.13 & $1.40[0.67 ; 2.95]$ & 0.38 & $4.90 * * *[1.33 ; 17.99]$ & 0.01 & $5.94 * * *[2.41 ; 14.63]$ & $<0.01$ \\
\hline$>10$ & $1.27[0.75 ; 2.14]$ & 0.38 & $1.67 *[0.82 ; 3.38]$ & 0.16 & $3.05[0.99 ; 9.38]$ & 0.04 & $4.06[2.01 ; 8.21]$ & $<0.01$ \\
\hline$>$ Optimal: treatment & $2.22 * * *[1.21 ; 4.07]$ & 0.01 & $2.87 * * *[1.25 ; 6.63]$ & 0.01 & $4.90 * * *[1.33 ; 17.99]$ & 0.01 & $5.43[2.34 ; 12.59]$ & $<0.01$ \\
\hline >Optimal & $1.64[0.97 ; 2.76]$ & 0.06 & $1.72[0.83 ; 3.60]$ & 0.15 & $4.90[1.33 ; 17.99]$ & 0.01 & $5.41[2.33 ; 12.55]$ & $<0.01$ \\
\hline
\end{tabular}

Prognostic value of baseline Chromogranin A (CgA) for cancer-specific (CSS) and progression-free survival (PFS) given as continuous values, at "standard" cut-offs $2 \times, 5 \times, 10 \times \mathrm{ULN}$ and at "optimal" estimated cut-offs for the whole cohort (optimal) and per treatment given (optimal: treatment). Significant $p$ values are marked in bold numbers. Hazard ratios providing best discrimination are marked with $* * *$ (all cut-offs) and with * for "standard" cut-offs, when different

$U L N$ upper limit of normal, SSA somatostatin analogues, IFN Interferon-alpha, PRRT peptide receptor radionuclide therapy, NA not available 
Table 3 Baseline 5HIAA as predictor for CSS, PFS

\begin{tabular}{|c|c|c|c|c|c|c|c|c|}
\hline & \multicolumn{2}{|l|}{ SSA single } & \multicolumn{2}{|l|}{ SSA combined } & \multicolumn{2}{|l|}{ IFN } & \multicolumn{2}{|l|}{ PRRT } \\
\hline & $\mathrm{HR}[95 \% \mathrm{CI}]$ & $p$ & $\mathrm{HR}[95 \% \mathrm{CI}]$ & $p$ & $\mathrm{HR}[95 \% \mathrm{CI}]$ & $p$ & $\mathrm{HR}[95 \% \mathrm{CI}]$ & $p$ \\
\hline \multicolumn{9}{|l|}{ CSS } \\
\hline & $N=75$ & & $N=48$ & & $N=26$ & & $N=92$ & \\
\hline Continuous & $1.014[0.989 ; 1.040]$ & 0.26 & $1.014[1.002 ; 1.026]$ & 0.02 & $1.064[1.013 ; 1.119]$ & 0.01 & $1.071[1.038 ; 1.104]$ & $<0.01$ \\
\hline $5-10 \times$ & & 0.07 & & 0.02 & & 0.17 & & 0.01 \\
\hline $5-10 x$ & $2.50[0.98 ; 6.36]$ & & $0.38[0.09 ; 1.69]$ & & $1.35[0.27 ; 6.73]$ & & $2.08[1.04 ; 4.15]$ & \\
\hline$>10$ & $2.03[0.92 ; 4.45]$ & & $2.01[0.96 ; 4.21]$ & & 3.19 [0.90;11.39] & & $2.60[1.39 ; 4.85]$ & \\
\hline$>2$ & $1.62[0.79 ; 3.31]$ & 0.18 & $1.37[0.59 ; 3.18]$ & 0.47 & $1.08[0.35 ; 3.33]$ & 0.89 & $1.49[0.73 ; 3.06]$ & 0.27 \\
\hline$>5$ & $2.18 *[1.10 ; 4.34]$ & 0.02 & $1.33[0.65 ; 2.72]$ & 0.43 & $2.20[0.71 ; 6.84]$ & 0.16 & $2.36 *[1.34 ; 4.17]$ & $<0.01$ \\
\hline$>10$ & $1.70[0.81 ; 3.60]$ & 0.16 & $2.42 * * *[1.18 ; 4.94]$ & 0.01 & $2.98 *[0.89 ; 9.97]$ & 0.06 & $1.97[1.15 ; 3.38]$ & 0.01 \\
\hline$>$ Optimal: treatment & $2.54 * * *[1.25 ; 5.18]$ & 0.01 & $1.08[0.51 ; 2.30]$ & 0.84 & $3.89 * * *[1.22 ; 12.40]$ & 0.01 & $6.30 * * *[3.24 ; 12.27]$ & $<0.01$ \\
\hline$>$ Optimal & $1.98[1.00 ; 3.95]$ & 0.05 & $1.50[0.73 ; 3.06]$ & 0.26 & $2.63[0.83 ; 8.32]$ & 0.09 & $2.57[1.48 ; 4.46]$ & $<0.01$ \\
\hline \multicolumn{9}{|l|}{ PFS } \\
\hline & $N=75$ & & $N=45$ & & $N=26$ & & $N=92$ & \\
\hline Continuous & $1.003[0.985 ; 1.022]$ & 0.74 & $1.001[0.985 ; 1.017]$ & 0.90 & $1.026[0.986 ; 1.067]$ & 0.21 & $1.048[1.012 ; 1.087]$ & 0.01 \\
\hline $5-10 x$ & & 0.05 & & 0.40 & & 0.26 & & 0.01 \\
\hline $5-10 x$ & $2.54[1.16 ; 5.55]$ & & $1.15[0.41 ; 3.22]$ & & $3.03[0.74 ; 12.45]$ & & $3.06[1.46 ; 6.40]$ & \\
\hline$>10$ & $1.33[0.72 ; 2.46]$ & & $1.65[0.79 ; 3.44]$ & & $1.88[0.47 ; 7.61]$ & & $1.97[0.99 ; 3.95]$ & \\
\hline$>2$ & $1.64 *[0.95 ; 2.81]$ & 0.07 & $1.29[0.56 ; 2.99]$ & 0.54 & $1.69[0.52 ; 5.54]$ & 0.38 & $1.86[0.73 ; 4.74]$ & 0.19 \\
\hline$>5$ & $1.59[0.93 ; 2.72]$ & 0.09 & $1.49[0.74 ; 2.97]$ & 0.26 & $2.32 *[0.74 ; 7.27]$ & 0.14 & $2.34 * * *[1.26 ; 4.36]$ & 0.01 \\
\hline$>10$ & $1.17[0.65 ; 2.12]$ & 0.60 & $1.59 * * *[0.80 ; 3.15]$ & 0.18 & $1.46[0.39 ; 5.46]$ & 0.57 & $1.30[0.72 ; 2.34]$ & 0.39 \\
\hline$>$ Optimal: treatment & $2.08 * * *[1.11 ; 3.90]$ & 0.02 & $1.35[0.63 ; 2.91]$ & 0.43 & $2.53 * * *[0.75 ; 8.54]$ & 0.12 & $2.34 * * *[1.26 ; 4.36]$ & 0.01 \\
\hline >Optimal & $1.55[0.90 ; 2.65]$ & 0.11 & $1.49[0.74 ; 2.97]$ & 0.26 & $1.63[0.49 ; 5.46]$ & 0.42 & $2.34 * * *[1.26 ; 4.36]$ & 0.01 \\
\hline
\end{tabular}

Prognostic value of baseline 5-hydroxyindoleacetic acid (5HIAA) for cancer-specific (CSS) and progression-free survival (PFS) given as continuous values, at cut-offs $2 \times, 5 \times, 10 \times \mathrm{ULN}$ and at "optimal" estimated cut-offs for the whole cohort (optimal) and per treatment given (optimal: treatment). Significant $p$ values are marked in bold numbers. Hazard ratios providing best discrimination are marked with $* * *$ (all cutoffs) and with * for "standard" cut-offs

$U L N$ upper limit of normal, SSA somatostatin analogues, IFN Interferon-alpha, PRRT peptide receptor radionuclide therapy

$2 \times, 5 \times, 10 \times \mathrm{ULN}[12,15,16,21]$ and at a trichotomized cut-off $(5-10 \times \mathrm{ULN})$, as well as at the estimated optimal cut-off points for $\mathrm{CgA}$ and 5HIAA. Results for each treatment given are summarized in Tables 2 and 3, and for all cases, irrespective of treatment, in Supplementary Table 1. Sensitivity, specificity, PPV and NPV are shown in Supplementary Table 2 .

\section{Somatostatin analogues (SSA)}

We focused on single SSA and PRRT, the two treatments that are most applicable in current clinical practice. For patients treated with SSA, the estimated optimal cut-offs for baseline CgA were $2 \times \mathrm{ULN}$ for both CSS and PFS (Table 4). Indeed, $\mathrm{CgA}$ dichotomized at $2 \times \mathrm{ULN}$ defines a cohort with worse CSS (HR 5.30, 95\% CI 1.85-15.21, $p<0.01$ ) and PFS (HR $2.22,95 \%$ CI $1.21-4.07, p=0.01)$. In both cases HRs are clearly higher compared to other cut-offs (Fig. 2). Trichotomizing $\mathrm{CgA}$ at $5-10 \times \mathrm{ULN}$ did not seem to provide any additional benefit.
Table 4 Optimal cut-offs $(\times \mathrm{ULN})$

\begin{tabular}{lccccc}
\hline & \multicolumn{2}{c}{ CgA } & & \multicolumn{2}{c}{ 5HIAA } \\
\cline { 2 - 3 } \cline { 5 - 6 } & CSS & PFS & & CSS & PFS \\
\hline Irrespective of treatment & $\mathbf{6}$ & $\mathbf{6}$ & $\mathbf{6}$ & $\mathbf{5}$ \\
SSA single & 2 & 2 & & 4 & 1 \\
SSA combined & 90 & 45 & & 17 & 6 \\
IFN & 32 & 5 & 8 & 3 \\
PRRT & 4 & 7 & & 18 & 5 \\
\hline
\end{tabular}

Estimated "optimal" cut-offs for Chromogranin A $(\mathrm{CgA})$ and 5-hydroxyindoleacetic acid (5HIAA) using the maximally selected rank statistics, for the cohort as a whole (in bold), irrespective of treatment, and per treatment given

$U L N$ upper limit of normal, SSA somatostatin analogues, IFN Interferon-alpha, PRRT peptide receptor radionuclide therapy, CSS cancer-specific survival, $P F S$ progression-free survival

The estimated optimal 5HIAA cut-offs were $4 \times$ ULN for CSS and $1 \times$ ULN for PFS. The optimal estimated cut-offs separate two groups with a moderate HR of $2.54(95 \% \mathrm{CI}$ 


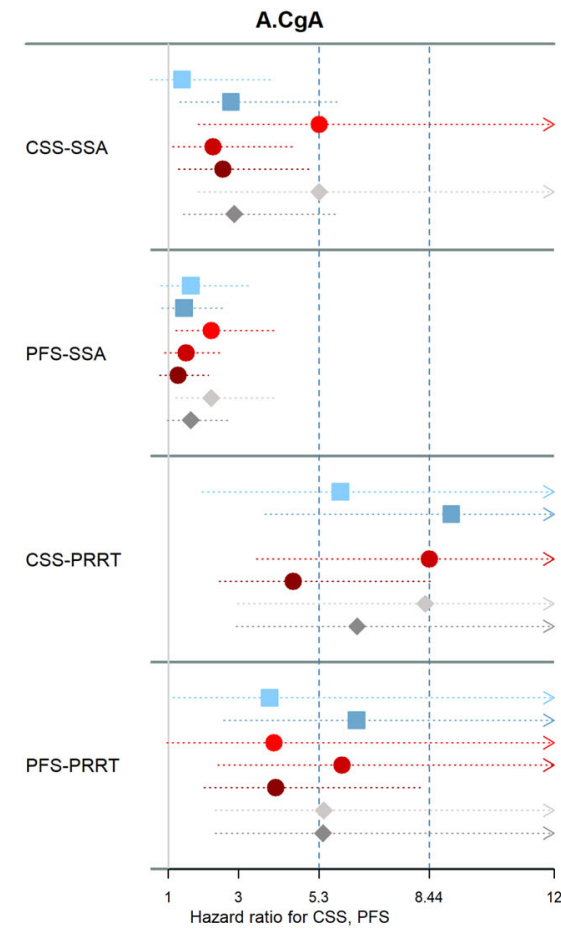

Fig. 2 Cancer-specific (CSS) and progression-free survival (PFS) for patients treated with somatostatin analogues (SSA) or peptide receptor radionuclide therapy (PRRT) at various cut-offs: trichotomized (squares), dichotomized (cycles) and at "optimal" estimated cut-offs (diamonds) for the whole cohort (opt: overall) and per treatment given (opt: treatment). A Chromogranin A ( $\mathrm{CgA})$, B 5-hydroxyindoleacetic acid (5HIAA). Dashed lines in place for "standard" cut-offs giving

$1.25-5.18, p=0.01)$ for CSS and 2.08 (95\% CI 1.11-3.90, $p=0.02$ ) for PFS (Table 3). These results, though statistically significant, are less discriminative in comparison to grouping by $\mathrm{CgA}$. A cut-off at $5 \times \mathrm{ULN}$ provides a slightly worse discrimination for CSS (HR 2.18, 95\% CI 1.10-4.34, $p=0.02$ ). Using two cut-off intervals at $5 \times$ and $10 \times \mathrm{ULN}$ unexpectedly resulted in lower HRs for the $>10 \times \mathrm{ULN}$ compared to the $5-10 \times \mathrm{ULN}$ group.

\section{Peptide receptor radionuclide therapy (PRRT)}

For patients treated with PRRT, the estimated optimal CgA cut-offs were $4 \times$ ULN for CSS and $7 \times$ ULN for PFS. Using two cut-offs at $5 \times$ and $10 \times$ ULN clearly distinguished three groups with HR of $5.90 \quad(95 \%$ CI 1.97-17.66) and 9.06 (95\% CI 3.75-21.88) for CSS, 3.88 (95\% CI 1.13-13.29) and 6.36 (95\% CI 2.58-15.72) for PFS. However, a single cut-off at $5 \times$ ULN provided similar discriminative value (HR 8.44, 95\% CI 3.51-20.31, $p<$ 0.01 for CSS and 5.94, 95\% CI 2.41-14.63, $p<0.01$ for PFS), is probably easier to use and provides higher HRs compared to other single cut-offs (Table 2 and Fig. 2).

The estimated optimal cut-offs for 5HIAA were $18 \times$ for CSS and $5 \times$ for PFS. As is the case with SSA, 5HIAA

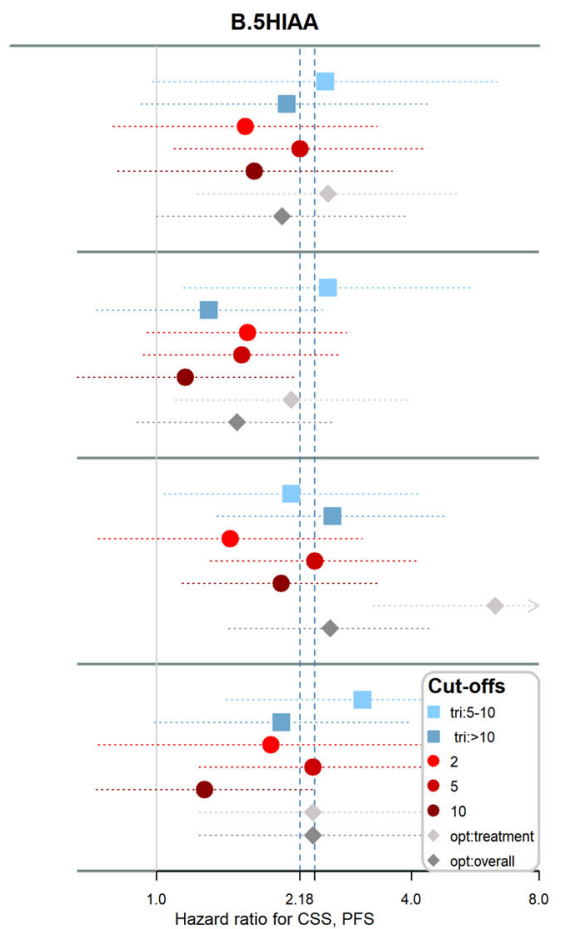

maximum discrimination for CSS. A CgA cut-off of $2 \times \mathrm{ULN}$ (upper limit of normal) seems to discriminate best for SSA, whereas $5 \times$ ULN and trichotomization discriminate well for PRRT. Discrimination by 5HIAA is lower irrespective of cut-off examined. In PRRT the "optimal" cut-off is clearly higher. The estimated "overall" optimal cut-offs (dark grey diamond) did not provide better discrimination in any case

provided worse discriminative value in comparison with $\mathrm{CgA}$, with similar HRs in the $2-10 \times \mathrm{ULN}$ range, which are more frequently used in the literature. The optimal HR in our cohort was provided at higher cut-off values for CSS, and discriminated sufficiently for CSS (HR 6.30, 95\% CI 3.24-12.27, $p<0.01$ ) and moderately for PFS (HR 2.34, $95 \%$ CI $1.26-4.36, p=0.01)$.

\section{Changes in biomarkers as prognostic markers of response}

\section{Somatostatin analogues (SSA)}

Biochemical test results at 6 months after treatment start were available for 98 (CgA) and 99 (5HIAA) patients among those who initially had a raised $\mathrm{CgA}$ and 5HIAA, respectively. The median reduction of $\mathrm{CgA}$ and 5HIAA were $43 \%$ and $41 \%$ respectively for all patients, $19 \%$ and $31 \%$ for patients starting treatment with single SSA, and $51 \%$ and $53 \%$ for patients starting a combination treatment (mostly with IFN and in nine cases with PRRT) at first line. Thirty-nine percent of all patients, $31 \%$ of patients treated with single SSA and $53 \%$ of patients starting on combined treatment achieved a reduction of $\mathrm{CgA}$ at 6 months of at 
least $50 \%$ and were classified as partial responders; the corresponding numbers for 5HIAA were 39\%, 31\% and $50 \%$.

Six-month reductions of $\mathrm{CgA}$ and 5HIAA correlated well with PFS and CSS for patients treated with single SSA, both as continuous and dichotomous variables (Table 5). It is worth noting that although CSS was reduced for patients progressing biochemically within 6 months of SSA start, the difference between patients having SD and those having PR was marginal (Fig. 3).

There were 133 events of escalation of SSA dose in 98 patients. The median change for $\mathrm{CgA}$ and 5HIAA was an increase of $17 \%$ and $15 \%$ respectively after the dose escalation. The biochemical response rate (RR) was $4.7 \%$ and $4.1 \%$ for $\mathrm{CgA}$ and 5HIAA, while $48 \%$ and $58 \%$, respectively, had SD at the 6-month control. Changes in $\mathrm{CgA}$ as a continuous variable showed a non-statistically significant correlation with CSS (HR 1.11, 95\% CI 0.99-1.25, $p=$ 0.08). There was no correlation between early 5HIAA changes as a continuous variable and either CSS or PFS. For both $\mathrm{CgA}$ and 5HIAA as dichotomous variables, there was no correlation with CSS (Table 5 and Fig. 3).

\section{Peptide receptor radionuclide therapy (PRRT)}

The median change of CgA and 5HIAA was a reduction of $15 \%$ and $12 \%$ respectively after PRRT, with RR of $18 \%$ and $18 \%$ for $\mathrm{CgA}$ and 5HIAA, while $66 \%$ and $67 \%$, respectively, had stable markers at the 6-month control. Reductions in CgA and in 5HIAA did not correlate with either PFS or CSS (Fig. 3). Results are summarized in Table 5.

Patients treated with PRRT were treated in parallel with various doses of SSA; Thirty-six percent of patients with available data were treated with up to standard doses $(30 \mathrm{mg}$ Sandostatin $^{\circledast}$ LAR /4 weeks or $120 \mathrm{mg}$ Somatuline ${ }^{\oplus}$ Autogel/4 weeks), $34 \%$ with SSA every 3 weeks and $30 \%$ with SSA every 2 weeks or higher doses. We examined whether different SSA doses could potentially mask the early effect of PRRT on CgA and 5HIAA levels stratifying for standard versus higher SSA doses. In this case early reductions of CgA had a borderline significant correlation with CSS (HR $1.45,95 \%$ CI $1.02-2.05, p=0.04)$, whereas early reductions of 5HIAA did not show any correlation (HR 1.29, 95\% CI 0.80-2.07, $p=0.29$ ). The correlation between early reduction of $\mathrm{CgA}$ and $\mathrm{CSS}$ remained significant even after correcting for baseline hormonal values, Ki-67 and performance status (Supplementary Table 3).

\section{Interferon-alpha (IFN)}

Of the 97 treatment cases with IFN during the study period, 58 had evaluable biomarkers both at baseline and after 6 months of treatment. Of those, $58 \%$ initiated treatment concomitantly, or shortly after, the initiation of $\mathrm{SSA}$, as was the clinical routine during the early study period. The median reduction of $\mathrm{CgA}$ and 5HIAA was $14 \%$ and $31 \%$ for the total population, with RR of $29 \%$ and $34 \%$. However, at least part of this effect will be due to the SSA analogue given concomitantly: For 35 patients starting treatment with IFN as single treatment, the median $\mathrm{CgA}$ increased by $5 \%$ and median 5HIAA decreased by $23 \%$ at 6 months from treatment start. Still, one out of seven patients had a more than $50 \%$ reduction of $\mathrm{CgA}$ and/or 5HIAA, and almost two thirds of evaluable patients had stabilization of those biochemical markers.

Because of the small number of patients, we examined patients having PD or SD together and compared them with responders; the groups were stratified by concomitant or not start of IFN. HR for CSS for responders vs. non-responders was statistically significant for 5HIAA (HR 2.59, 95\% CI $1.04-6.43, p=0.04$ ) but not for CgA (HR 1.91, 95\% CI $0.80-4.56, p=0.14)$. However only three of 19 evaluable responders were in the IFN single group and this result should be interpreted with caution.

\section{Changes in biomarkers and early change of treatment}

There were 83 cases of early biomarker progression: 23 on SSA, 11 on IFN, 16 on PRRT and 33 after SSA dose escalation. In 31 cases both $\mathrm{CgA}$ and 5HIAA increased by $>25 \%$, in 25 cases only $\mathrm{CgA}$ and in 27 cases only 5HIAA increased. Thirty-nine of those patients changed treatment within a year. In univariate analysis, CSS from treatment start did not differ significantly for those changing treatment early compared to patients who changed treatment more than a year later (HR 1.16, 95\% CI 0.67-2.00, $p=0.59$ ). However, it is likely that patients changing treatment early had a more aggressive disease and higher baseline hormone levels. In a multivariate analysis for baseline factors associated with more aggressive outcomes (CgA and 5HIAA at baseline, age, Ki-67), HR changed in favour of patients starting escalating their treatments earlier, but this was not statistically significant (HR $0.70,95 \%$ CI $0.37-1.33$, $p=0.28$ ).

\section{Discussion}

The present study examined the prognostic and predictive ability of two widely used biomarkers in clinical routine, plasma $\mathrm{CgA}$ and urine 5HIAA, focusing on their baseline values and relatively early changes during treatment. Unlike most previous studies that included various locations and histopathological grades of NET, it focused on a uniform 


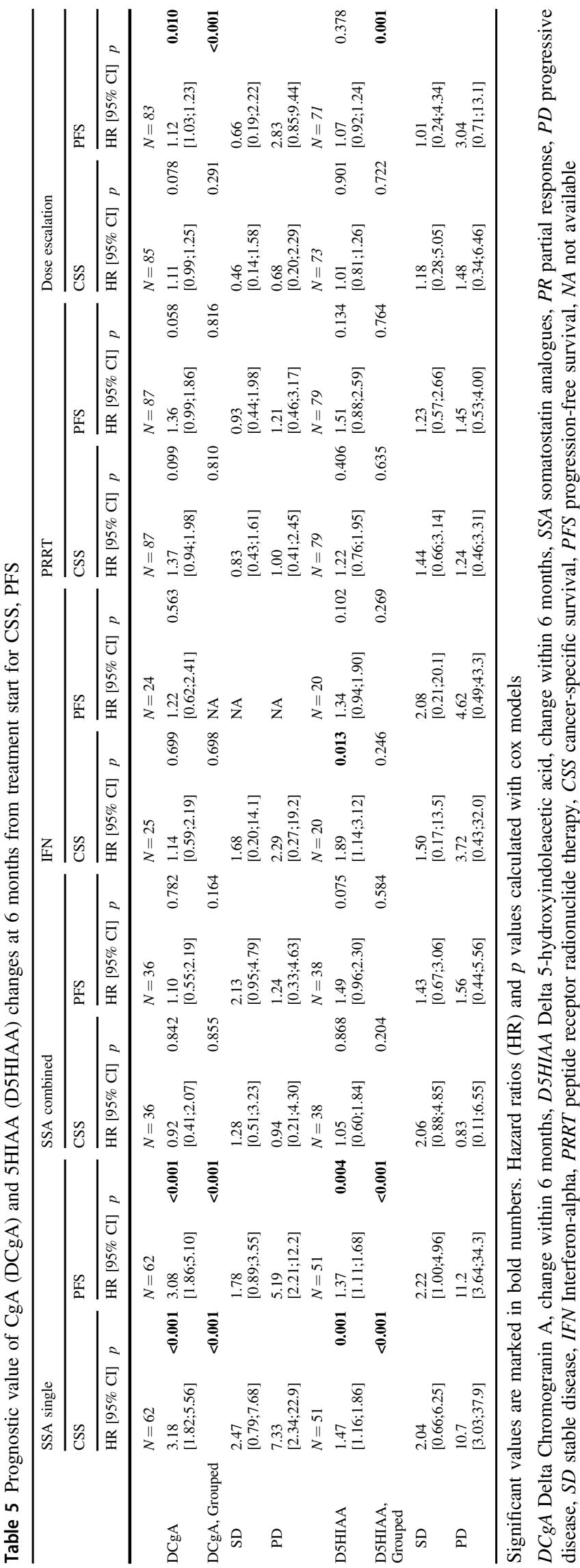

cohort of metastatic siNET G2 patients. The prognostic value of $\mathrm{CgA}$ and 5HIAA for the three major medical treatments used during the study period was examined. It was found that the level of baseline $\mathrm{CgA}$ and in certain cases 5HIAA are prognostic of CSS irrespective of treatment modality, and of PFS in patients treated with PRRT and possibly IFN. Changes of CgA and 5HIAA levels at 6 months of treatment correlate well with CSS and PFS in patients treated with SSA, but not in patients treated with PRRT. The number of patients treated with single IFN was too small to draw any definite conclusions.

Elevated baseline $\mathrm{CgA}$ levels have long been considered as markers of poor outcome. Indeed, higher $\mathrm{CgA}$ levels correlate with high metastatic load [22-24], and with poorer survival in the metastatic setting [22, 24]. Prospective analyses of the RADIANT trials provide evidence of a shorter survival in patients with elevated baseline $\mathrm{CgA}$, using a cut-off of $2 \times$ ULN $[12,15]$. The same cut-off was used in a recently published randomized trial comparing interferon with bevacizumab [8]. Other authors have used cut-offs ranging from $1 \times$ to $10 \times \mathrm{ULN}[7,10,11,13,14]$, or even studied $\mathrm{CgA}$ levels divided into three groups [16, 25]. We examined three different cut-offs used in the literature $(2 \times, 5 \times$ and $10 \times$ ULN), as well as "optimal" cut-offs. In our cohort, a standard cut-off of $2 \times$ ULN presents a better discriminative value in patients treated with SSA, whereas $5 \times$ ULN seems more appropriate in combination with PRRT and probably IFN. The estimated "optimal" cut-offs presented only marginal, if any, advantage over those routinely used in the literature.

A recently published prospective study showed only a weak association between changes of $\mathrm{CgA}$ and changes in tumour burden [26]. Post hoc analysis from phase 3 trials showed that an early decrease in $\mathrm{CgA}$ related to a decreased risk of PD for SSA [18] and everolimus [17] but not for PRRT [14]. A post hoc analysis of the NETTER-1 phase 3 trial comparing PRRT with high-dose SSA showed that there was a statistically significant correlation between 6-month radiological response and PFS for patients treated with SSA but not with PRRT [27]. It is also worth noting that in the case of another well-studied radionuclide used in the treatment of metastatic prostate cancer, Radium-223, changes of the tumour marker prostate-specific antigen is not considered a reliable surrogate marker for survival [28]. These results are largely consistent with our study findings. All trials above used mixed study populations, with less than half of the study population having siNETs. Two recent reviews of $\mathrm{CgA}$ as a biomarker reached somewhat contradicting conclusions: Both agree that higher baseline $\mathrm{CgA}$ is associated with shorter PFS. When it comes to early response evaluation, the first review indicates that an early response is associated with better clinical outcomes [29] and the second that circulating $\mathrm{CgA}$ does not represent a valid 

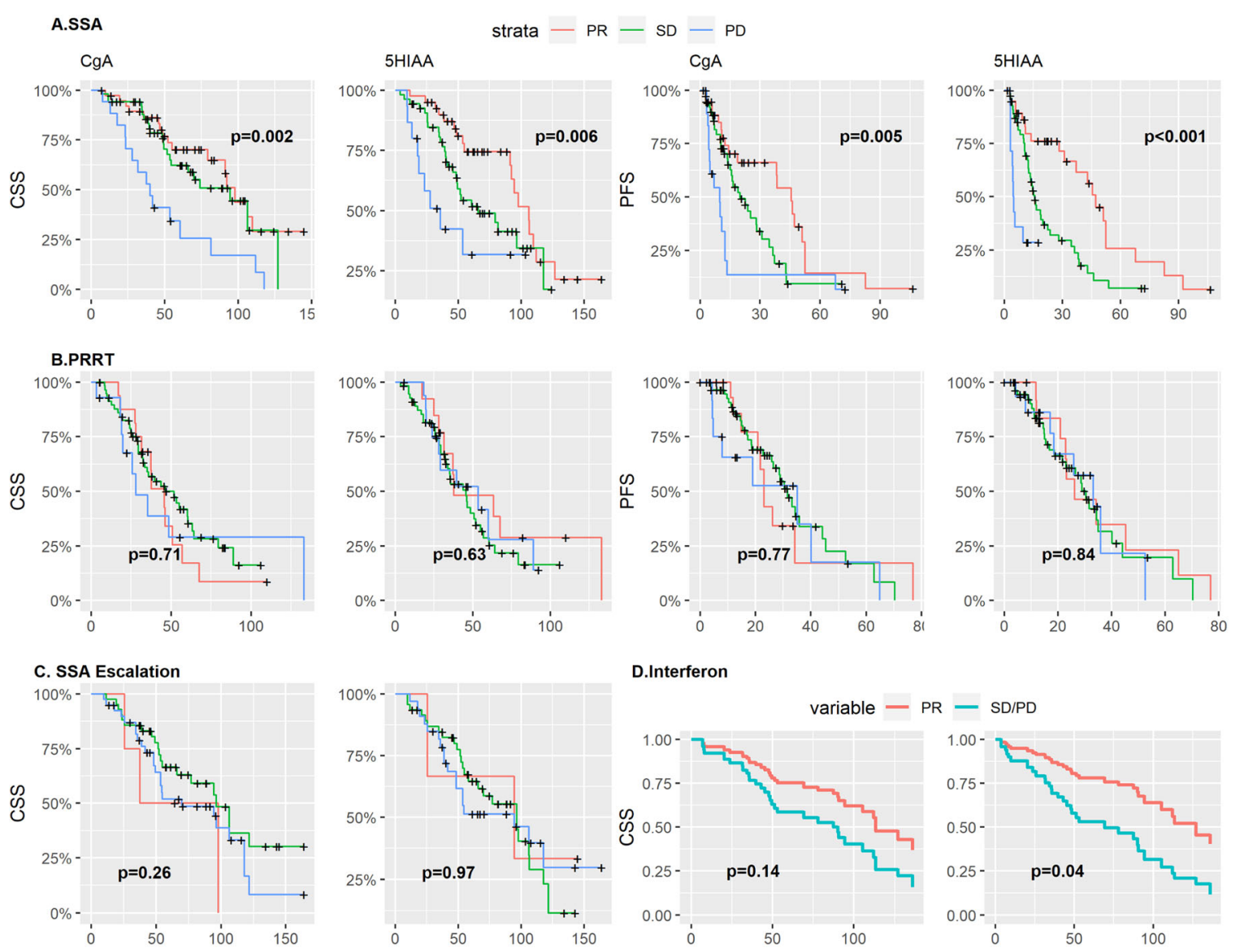

D.Interferon

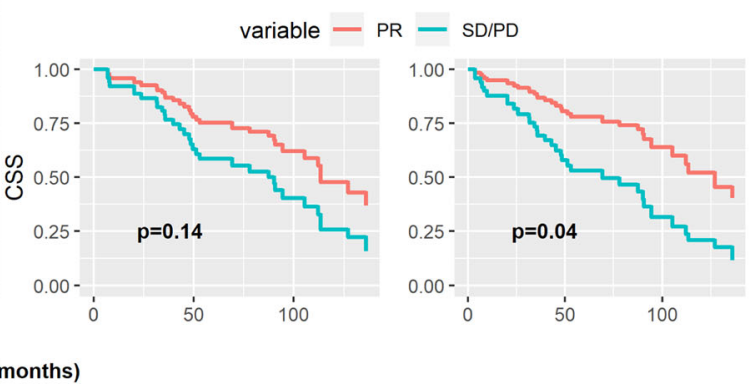

Fig. 3 Cancer-specific survival (CSS) and progression-free survival (PFS) after treatment with somatostatin analogues (SSA) (A), peptide receptor radionuclide therapy (PRRT) (B) and SSA dose escalation (C) stratified by 6 -month biochemical response. Adjusted survival curves for CSS in patients treated with interferon, as a function of

biochemical response for the cox model, stratified for concomitant use of SSA (D). CgA Chromogranin A, 5HIAA 5-hydroxyindoleacetic acid, PR partial response, SD stable disease, PD progressive disease. $P$ values derived from log-rank test $(\mathbf{A}-\mathbf{C})$ and cox models $(\mathbf{D})$

marker of morphological evolution of disease and has therefore no utility in this setting [30].

Less is known about the prognostic and predictive value of 5HIAA in siNETs. Zandee et al. reported that baseline 5HIAA at a cut-off of $10 \times \mathrm{ULN}$ is a negative prognostic factor of overall survival (OS) in univariate, but not multivariate analysis [25]. On the other hand, Laskaratos et al. reported that 5HIAA at a cut-off of $10 \times \mathrm{ULN}$ together with age were the only factors that remain significant in multivariate analysis for OS of siNETs with desmoplasia [16]. Data about 5HIAA changes associated with specific treatments are limited. In our study, 5HIAA seems to be prognostic of PFS and CSS in patients treated with PRRT, but not with single SSA. It is also significant as a prognostic factor for CSS for patients treated with interferon. The optimal cut-off is higher than that for $\mathrm{CgA}$ but varies between treatments, and HRs are generally similar in the range of $2-10 \times$ ULN. At least in the case of PRRT the optimal cut-off for CSS might be significantly higher and in our cohort it was $18 \times$ ULN.

We examined early biomarker changes in some specific situations: First we showed that in a group of patients treated with escalated doses of SSA, early continuous decreases of $\mathrm{CgA}$, but not 5HIAA, had borderline correlation with CSS and PFS. Moreover, we hypothesized that early biomarker changes in patients treated with PRRT might be masked by concomitant treatment with SSA. Unfortunately, we could not examine biomarker changes in an SSA-naive population, but we examined differences depending on the different baseline SSA doses. Early changes of $\mathrm{CgA}$ but not 5HIAA showed a borderline correlation with CSS after correcting for baseline SSA doses.

As more treatment options become available, it is important to investigate if early intensification of treatment can improve survival. Approximately half of the patients with early biochemical progression changed treatment within a year of treatment start. We compared patients with early treatment changes with those who remained on the same treatment for a longer time. In 
univariate analysis there was no difference in CSS (HR 1.16, 95\% CI: $0.67-2.00, p=0.59$ ), but the HR switched in favour of early treatment changes when adjusting for known prognostic factors of poor response in a multivariate analysis (HR $0.70,95 \%$ CI $0.37-1.33, p=0.28$ ). A larger study could give a definitive answer to this question.

Monoanalytes, such as $\mathrm{CgA}$ and 5HIAA have been criticized for lacking specificity and sensitivity [31, 32]. Genomic assays such as the NETest ${ }^{\circ}$ have been argued to provide a more precise alternative [32]. However, trials to date have examined the prognostic ability of the NETest ${ }^{\circ}$ in relation to disease status and progression instead of OS. Besides, genomic tests have struggled to become mainstream in most oncologic fields and are almost never used in the metastatic setting: For example the 15-year-old Oncotype $\mathrm{DX}^{\odot}$ in breast cancer still had $35 \%$ adoption rate among US physicians and as low as $<20 \%$ in some areas in Europe [33, 34]. Simple monoanalytes are still used routinely in several solid tumours and, despite their limitations, are probably unlikely to be completely replaced by multigenomic assays in the very near future.

The study has several limitations. First, the study population is mostly that of a tertiary referral centre, and might not be representative of the general population. Second, this is a retrospective study with laboratory tests spanning over a 20 -year period and there is considerable variation between different methods of measuring $\mathrm{CgA}$ and 5HIAA. However, more than two-thirds of tests in SSA and IFN patients and virtually all tests in PRRT patients were conducted in a single reference laboratory. Finally, treatment patterns changed during the study period; most notably IFN concomitantly with SSA as a first line treatment is rarely used nowadays, and the IFN results might not be applicable to patients treated with IFN at second or later lines.

In conclusion, we have shown that $\mathrm{CgA}$ and to a lesser extent 5HIAA baseline levels are associated with CSS in patients with siNETs G2, irrespective of treatment used, and with PFS in patients treated with PRRT and we suggest optimal cut-off points for dichotomizing those variables. The reductions of $\mathrm{CgA}$ and 5HIAA at 6 months from treatment start have prognostic utility in patients treated with SSA, but not in patients treated with PRRT.

Funding This study was supported by the Swedish Cancer Society (grant number 18 0576) and Futurum-the Academy for Health and Care, Region Jönköping County. Open access funding provided by Uppsala University.

\section{Compliance with ethical standards}

Conflict of interest The authors declare that they have no conflict of interest.
Ethical approval This study has been approved by the Uppsala ethical review board (Dnr 2017/403).

Informed consent Patients treated at the Department of endocrine oncology, Uppsala university hospital, have provided written consent at time of treatment for the use of clinical data in retrospective research projects. Alive patients treated externally have provided written informed consent when included to the study. The Uppsala ethical review board has waived the need for written consent for the minority of already deceased patients that have been treated externally and where written consent was not previously provided.

Publisher's note Springer Nature remains neutral with regard to jurisdictional claims in published maps and institutional affiliations.

Open Access This article is licensed under a Creative Commons Attribution 4.0 International License, which permits use, sharing, adaptation, distribution and reproduction in any medium or format, as long as you give appropriate credit to the original author(s) and the source, provide a link to the Creative Commons license, and indicate if changes were made. The images or other third party material in this article are included in the article's Creative Commons license, unless indicated otherwise in a credit line to the material. If material is not included in the article's Creative Commons license and your intended use is not permitted by statutory regulation or exceeds the permitted use, you will need to obtain permission directly from the copyright holder. To view a copy of this license, visit http://creativecommons. org/licenses/by/4.0/.

\section{References}

1. B. Niederle, U.-F. Pape, F. Costa, D. Gross, F. Kelestimur, U. Knigge, K. Öberg, M. Pavel, A. Perren, C. Toumpanakis, J. O"Connor, D. O'Toole, E. Krenning, N. Reed, R. Kianmanesh; Participants, all other V.C.C., ENETS consensus guidelines update for neuroendocrine neoplasms of the jejunum and ileum. Neuroendocrinology 103, 125-138 (2016). https://doi.org/10. $1159 / 000443170$

2. D. Klimstra, G. Kloppell, S. Rosa La, G. Rindi, Classification of neuroendocrine neoplasms of the digestive system. WHO classification of tumours: digestive system tumours, 5th ed, WHO Classification of Tumours Editorial Board (International Agency for Research on Cancer, Lyon, 2019)

3. D. Man, J. Wu, Z. Shen, X. Zhu, Prognosis of patients with neuroendocrine tumor: a SEER database analysis. Cancer Manag. Res. 10, 5629-5638 (2018). https://doi.org/10.2147/CMAR.S174907

4. E. Andersson, Y. Arvidsson, C. Swärd, T. Hofving, B. Wängberg, E. Kristiansson, O. Nilsson, Expression profiling of small intestinal neuroendocrine tumors identifies subgroups with clinical relevance, prognostic markers and therapeutic targets. Mod. Pathol. 29, 616-629 (2016). https://doi.org/10.1038/modpathol.2016.48

5. V. Marotta, V. Nuzzo, T. Ferrara, A. Zuccoli, M. Masone, L. Nocerino, M.D. Prete, F. Marciello, V. Ramundo, G. Lombardi, M. itale, A. Colao, A. Faggiano, Limitations of chromogranin A in clinical practice. Biomark. Biochem. Indic. Expo. Response Susceptibility Chem. 17, 186-191 (2012). https://doi.org/10.3109/ 1354750X.2012.654511

6. K. Oberg, I.M. Modlin, W. De Herder, M. Pavel, D. Klimstra, A. Frilling, D.C. Metz, A. Heaney, D. Kwekkeboom, J. Strosberg, T. Meyer, S.F. Moss, K. Washington, E. Wolin, E. Liu, J. Goldenring, Consensus on biomarkers for neuroendocrine tumour disease. Lancet Oncol. 16, e435-e446 (2015). https://doi.org/10. 1016/S1470-2045(15)00186-2 
7. W. Rogowski, E. Wachuła, A. Lewczuk, A. Kolesińska-Ćwikła, E. Iżycka-Świeszewska, V. Sulżyc-Bielicka, J.B. Ćwikła, Baseline chromogranin A and its dynamics are prognostic markers in gastroenteropancreatic neuroendocrine tumors. Future Oncol. Lond. Engl. 13, 1069-1079 (2017). https://doi.org/10.2217/fon-2016-0455

8. J.C. Yao, K.A. Guthrie, C. Moran, J.R. Strosberg, M.H. Kulke, J.A. Chan, N. LoConte, R.R. McWilliams, E.M. Wolin, B. Mattar, S. McDonough, H. Chen, C.D. Blanke, H.S. Hochster, Phase III prospective randomized comparison trial of depot octreotide plus interferon alfa- $2 \mathrm{~b}$ versus depot octreotide plus bevacizumab in patients with advanced carcinoid tumors: SWOG S0518. J. Clin. Oncol. 35, 1695-1703 (2017). https://doi.org/10.1200/JCO.2016.70.4072

9. F.-M. Laskaratos, M. Walker, D. Wilkins, A. Tuck, S. Ramakrishnan, E. Phillips, J. Gertner, M. Megapanou, D. Papantoniou, R. Shah, J. Banks, E. Vlachou, J. Garcia-Hernandez, L. Woodbridge, A. Papadopoulou, L. Grant, E. Theocharidou, J. Watkins, T.V. Luong, D. Mandair, M. Caplin, C. Toumpanakis, Evaluation of clinical prognostic factors and further delineation of the effect of mesenteric fibrosis on survival in advanced midgut neuroendocrine tumours. Neuroendocrinology 107, 292-304 (2018). https://doi.org/10.1159/000493317

10. S. Ekeblad, B. Skogseid, K. Dunder, K. Öberg, B. Eriksson, Prognostic factors and survival in 324 patients with pancreatic endocrine tumor treated at a single institution. Clin. Cancer Res. 14, 7798-7803 (2008). https://doi.org/10.1158/1078-0432.CCR08-0734

11. I.M. Modlin, B.I. Gustafsson, M. Pavel, B. Svejda, B. Lawrence, M. Kidd, A nomogram to assess small-intestinal neuroendocrine tumor ('Carcinoid') survival. Neuroendocrinology 92, 143-157 (2010). https://doi.org/10.1159/000319784

12. J.C. Yao, M. Pavel, A.T. Phan, M.H. Kulke, S. Hoosen, J. St. Peter, A. Cherfi, K.E. Öberg, Chromogranin A and neuronspecific enolase as prognostic markers in patients with advanced pNET treated with everolimus. J. Clin. Endocrinol. Metab. 96, 3741-3749 (2011). https://doi.org/10.1210/jc.2011-0666

13. M. Ter-Minassian, J.A. Chan, S.M. Hooshmand, L.K. Brais, A. Daskalova, R. Heafield, L. Buchanan, Z.R. Qian, C.S. Fuchs, X. Lin, D.C. Christiani, M.H. Kulke, Clinical presentation, recurrence, and survival in patients with neuroendocrine tumors: results from a prospective institutional database. Endocr. Relat. Cancer. 20, 187-196 (2013). https://doi.org/10.1530/ERC-12-0340

14. L. Bodei, M. Kidd, I.M. Modlin, S. Severi, I. Drozdov, S. Nicolini, D.J. Kwekkeboom, E.P. Krenning, R.P. Baum, G. Paganelli, Measurement of circulating transcripts and gene cluster analysis predicts and defines therapeutic efficacy of peptide receptor radionuclide therapy (PRRT) in neuroendocrine tumors. Eur. J. Nucl. Med. Mol. Imaging. 43, 839-851 (2016). https://doi.org/10. 1007/s00259-015-3250-z

15. M.E. Pavel, E. Baudin, K.E. Öberg, J.D. Hainsworth, M. Voi, N. Rouyrre, M. Peeters, D.J. Gross, J.C. Yao, Efficacy of everolimus plus octreotide LAR in patients with advanced neuroendocrine tumor and carcinoid syndrome: final overall survival from the randomized, placebo-controlled phase 3 RADIANT-2 study. Ann. Oncol. 28, 1569-1575 (2017). https://doi.org/10.1093/annonc/mdx193

16. F.M. Laskaratos, L. Diamantopoulos, M. Walker, H. Walton, M. Khalifa, F. El-Khouly, A. Koffas, G. Demetriou, M. Caplin, C. Toumpanakis, D. Mandair, Prognostic factors for survival among patients with small bowel neuroendocrine tumours associated with mesenteric desmoplasia. Neuroendocrinology 106, 366-380 (2018). https://doi.org/10.1159/000486097

17. E. Baudin, E. Wolin, D. Castellano, G. Kaltsas, A. Panneerselvam, Z. Tsuchihashi, S. Saletan, J.C. Yao, D. Gross, 6564 POSTER correlation of PFS with early response of chromogranin A and 5hydroxyindoleacetic acid levels in Pts with advanced neuroendocrine tumours: phase III RADIANT-2 study results. Eur. J. Cancer 47, S460 (2011). https://doi.org/10.1016/S0959-8049(11)71875-5
18. N. Buil-Bruna, M. Dehez, A. Manon, T.X.Q. Nguyen, I.F. Trocóniz, Establishing the quantitative relationship between lanreotide Autogel $^{\circledast}$, chromogranin A, and progression-free survival in patients with nonfunctioning gastroenteropancreatic neuroendocrine tumors. AAPS J. 18, 703-712 (2016). https://doi.org/10. 1208/s12248-016-9884-3

19. T. Hothorn, B. Lausen, On the exact distribution of maximally selected rank statistics. Social Science Research Network, Rochester, NY (2002)

20. T. Hothorn, B. Lausen, Maximally selected rank statistics in R. https://cran.r-project.org/web/packages/maxstat/vignettes/maxstat. pdf. Accessed 29 Feb 2020

21. J.R. Strosberg, J.M. Weber, M. Feldman, D. Coppola, K. Meredith, L.K. Kvols, Prognostic validity of the American Joint Committee on Cancer staging classification for midgut neuroendocrine tumors. J. Clin. Oncol. 31, 420-425 (2013). https:// doi.org/10.1200/JCO.2012.44.5924

22. E.T. Janson, L. Holmberg, M. Stridsberg, B. Eriksson, E. Theodorsson, E. Wilander, K. Öberg, Carcinoid tumors: analysis of prognostic factors and survival in 301 patients from a referral center. Ann. Oncol. 8, 685-690 (1997). https://doi.org/10.1023/A: 1008215730767

23. D. Campana, F. Nori, L. Piscitelli, A.M. Morselli-Labate, R. Pezzilli, R. Corinaldesi, P. Tomassetti, Chromogranin A: is it a useful marker of neuroendocrine tumors? J. Clin. Oncol. 25, 1967-1973 (2007). https://doi.org/10.1200/JCO.2006.10.1535

24. G.C. Nikou, K. Marinou, P. Thomakos, D. Papageorgiou, V. Sanzanidis, P. Nikolaou, C. Kosmidis, A. Moulakakis, E. Mallas, Chromogranin a levels in diagnosis, treatment and follow-up of 42 patients with non-functioning pancreatic endocrine tumours. Pancreatology 8, 510-519 (2008). https://doi.org/10.1159/000152000

25. W.T. Zandee, K. Kamp, R.C.S. Adrichem, R.A. van, Feelders, W. W. de Herder, Limited value for urinary 5-HIAA excretion as prognostic marker in gastrointestinal neuroendocrine tumours. Eur. J. Endocrinol. 175, 361-366 (2016). https://doi.org/10.1530/ EJE-16-0392

26. G. Dam, H. Grønbæk, H. Sorbye, E. Thiis Evensen, B. Paulsson, A. Sundin, C. Jensen, D. Ebbesen, U. Knigge, E. Tiensuu Janson, Prospective study of chromogranin A as a predictor of progression in patients with pancreatic, small-intestinal, and unknown primary neuroendocrine tumors. Neuroendocrinology 110, 217-224 (2020). https://doi.org/10.1159/000503833

27. M.E. Pavel, P. Broberg, M. Caplin, P. Ruszniewski, J. Strosberg, P. Santoro, L. Ravasi, E. Krenning, 1382PDRelation between objective tumour shrinkage and progression-free survival (PFS) in the NETTER-1 population. Ann. Oncol. 30 (2019). https://doi.org/ 10.1093/annonc/mdz256.002

28. D. Heinrich, J. Bektic, A.M. Bergman, O. Caffo, R. Cathomas, K. N. Chi, G. Daugaard, D. Keizman, J. Kindblom, G. Kramer, D. Olmos, A. Omlin, S.S. Sridhar, M. Tucci, I. van Oort, S. Nilsson, The contemporary use of Radium-223 in metastatic castrationresistant prostate cancer. Clin. Genitourin. Cancer 16, e223-e231 (2018). https://doi.org/10.1016/j.clgc.2017.08.020

29. P. Di Giacinto, F. Rota, L. Rizza, D. Campana, A. Isidori, A. Lania, A. Lenzi, P. Zuppi, R. Baldelli, Chromogranin A.: from laboratory to clinical aspects of patients with neuroendocrine tumors. Int. J. Endocrinol. 2018 (2018). https://doi.org/10.1155/ 2018/8126087

30. V. Marotta, M.C. Zatelli, C. Sciammarella, M.R. Ambrosio, M. Bondanelli, A. Colao, A. Faggiano, Chromogranin A as circulating marker for diagnosis and management of neuroendocrine neoplasms: more flaws than fame. Endocr. Relat. Cancer 25, R11-R29 (2018). https://doi.org/10.1530/ERC-17-0269

31. I.M. Modlin, I. Drozdov, D. Alaimo, S. Callahan, N. Teixiera, L. Bodei, M. Kidd, A multianalyte PCR blood test outperforms single analyte ELISAs (chromogranin A, pancreastatin, 
neurokinin A) for neuroendocrine tumor detection. Endocr. Relat. Cancer 21, 615-628 (2014). https://doi.org/10.1530/ERC-140190

32. Öberg, K. Molecular genomic blood biomarkers for neuroendocrine tumors: the long and winding road from Berzelius and Bence Jones to a neuroendocrine destination. Neuroendocrinology (2020). https://doi.org/10.1159/000508488

33. M.S. Aapro, M.D. Laurentiis, E. Mamounas, M. Martin, D. Rea, R. Rouzier, V. Smit, C. Thomssen, Adoption of multi-gene assays in HR +, HER2 - breast cancer (BC) patients in Europe: results of the Multidisciplinary Application of Genomics in Clinical Practice (MAGIC) survey. Ann. Oncol. 25, i5 (2014). https://doi.org/10. 1093/annonc/mdu065.3

34. S.C. O’Neill, C. Isaacs, C. Chao, H.-T. Tsai, C. Liu, B.F. Ekezue, N. Selvam, L.G. Kessler, M.D. Schwartz, T. Lobo, A.L. Potosky, Adoption of gene expression profiling for breast cancer in US oncology practice for women under age 65. J. Natl Compr. Cancer Netw. 13, 1216-1224 (2015) 